\title{
Temperature dependence of the breakdown of the quantum Hall effect studied by induced currents
}

\author{
A. J. Matthews, K. V. Kavokin,* A. Usher, M. E. Portnoi, M. Zhu, and J. D. Gething \\ Quantum Interacting Systems Group, School of Physics, University of Exeter, Stocker Road, Exeter EX4 4QL, United Kingdom \\ M. Elliott, W. G. Herrenden-Harker, and K. Phillips \\ School of Physics and Astronomy, Cardiff University, P.O. Box 913, Cardiff CF24 3YB, United Kingdom \\ D. A. Ritchie and M. Y. Simmons ${ }^{\dagger}$ \\ Cavendish Laboratory, University of Cambridge, Madingley Road, Cambridge CB3 OHE, United Kingdom \\ C. B. Sorensen and O. P. Hansen \\ Niels Bohr Institute Ørsted Laboratory, Universitetsparken 5, DK-2100 Copenhagen Ø, Denmark \\ O. A. Mironov, M. Myronov, and D. R. Leadley \\ Department of Physics, University of Warwick, Gibbet Hill Road, Coventry CV4 7AL, United Kingdom \\ M. Henini \\ School of Physics and Astronomy, University of Nottingham, Nottingham NG7 2RD, United Kingdom \\ (Received 11 October 2003; revised manuscript received 15 March 2004; published 31 August 2004)
}

\begin{abstract}
We have developed a model of the high-current breakdown of the integer quantum Hall effect, as measured in contactless experiments using a highly-sensitive torsion balance magnetometer. The model predicts that, for empirically "low-mobility" samples $\left(\mu<75 \mathrm{~m}^{2} \mathrm{~V}^{-1} \mathrm{~s}^{-1}\right)$, the critical current for breakdown should decrease with, and have a linear dependence on, temperature. This prediction is verified experimentally with the addition of a low-temperature saturation of the critical current at a temperature that depends on both sample number density and filling factor. It is shown that this saturation is consistent with quasielastic inter-Landau-level scattering when the maximum electric field in the sample reaches a large enough value. In addition we show how this model can be extended to give qualitative agreement with experiments on high-mobility samples.

DOI: 10.1103/PhysRevB.70.075317

PACS number(s): 73.43. $-\mathrm{f}$
\end{abstract}

\section{INTRODUCTION}

Shortly after the discovery of the integer quantum hall effect (IQHE), ${ }^{1}$ in transport measurements on twodimensional electron systems (2DESs) using Hall bar geometry, it was found that the associated dissipationless state, $\rho_{x x}=0$, would break down if a sufficiently large current was passed through the device. ${ }^{2}$ These early measurements ascribed a critical current density of approximately $0.5 \mathrm{~A} \mathrm{~m}^{-1}$, corresponding to a critical Hall electric field of the order of $10^{4} \mathrm{~V} \mathrm{~m}^{-1}$. Later work on Hall bars with narrow constrictions ${ }^{3}$ gave much higher critical fields of the order of $10^{5} \mathrm{~V} \mathrm{~m}^{-1}$. There have been several theories put forward to explain the breakdown of the IQHE (Refs. 4-6) but, as yet, there is no consensus.

There have also been detailed transport-experiment studies into the IQHE. Most of these measurements have been made on samples with Hall bar geometry ${ }^{7}$ but some have utilised Corbino devices. ${ }^{8}$ A different experimental approach was employed by Dolgopolov et al., ${ }^{9}$ here a Corbino device was subjected to a time-varying magnetic field. As the field was swept charge transfer was measured between the inner and outer contact using an electrometer and, as expected, their signal was hysteretic-reversing sign when the direction of the magnetic field sweep was changed. Recent experimental work has shown that sample geometry, mobility, and contact configuration can all affect the measured critical current. ${ }^{10}$ For this reason, a contactless geometry is desirable for IQHE breakdown measurements.

An experimental method with which contactless IQHE breakdown measurements may be performed was developed by Jones et al. ${ }^{11}$ whereby the currents induced in a 2DES by subjecting it to a sweeping magnetic field are detected using a sensitive torsion balance magnetometer. In this letter we develop a model, based on an original idea by Dyakonov, ${ }^{12}$ to show that such induced currents are sustained by charge transfer throughout the bulk of the 2DES sample. This model, for the first time, allows the very strong temperature dependence of these induced currents to be explained and analysis of new experimental data from a variety of samples and over a range of Landau level filling factors allows for information about $g$-factors, the density of states between Landau levels and the breakdown of the IQHE at high currents to be extracted.

\section{EXPERIMENTAL DETAILS}

Currents are induced in a 2DES by subjecting it to a timevarying magnetic field. These induced eddy currents (ECs) become large as $\rho_{x x}$ tends to zero at integer, and some fractional, values of the Landau level (LL) filling factor, $\nu$, and 


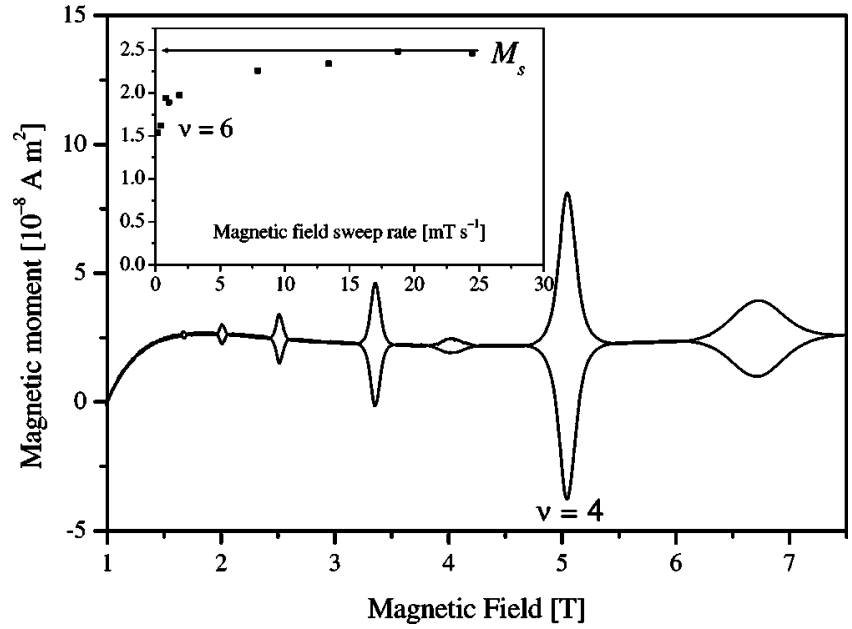

FIG. 1. Raw experimental data showing induced currents, as their corresponding magnetic moment, in sample $\mathrm{T} 73$ at $50 \mathrm{mK}$. There is a slowly varying background arising from the magnetization of the rotor. The induced currents can clearly be seen as they reverse polarity when the field sweep direction is reversed. The inset shows an example of a $M$ vs $\partial B / \partial t$ curve for $\nu=6$ at $50 \mathrm{mK}$, the size of the induced current saturates at fast sweep rates and the value of $M_{s}$ is indicated.

can be detected via their associated magnetic moment, $M$, using a torsion balance magnetometer. ${ }^{11}$

The samples were mounted on the torque magnetometer, which has been described elsewhere, ${ }^{13}$ so that the normal to the plane of the 2DES was at an angle of $20^{\circ}$ to the applied magnetic field. The magnetometer was placed in the mixing chamber of a dilution refrigerator, in the dilute phase, and the whole assembly inserted into the bore of a superconducting solenoid magnet. One of the dilution units used in this work has a base temperature of $50 \mathrm{mK}$ and the other has a base temperature of below $10 \mathrm{mK}$. The magnetic field was swept at rates from approximately $2 \times 10^{-4} \mathrm{~T} \mathrm{~s}^{-1}$ to $2 \times 10^{-2} \mathrm{~T} \mathrm{~s}^{-1}$ covering a range of $0-19 \mathrm{~T}$. In situ calibration of the device is possible by passing a known current through a coil, wound around a solid former, which is attached to the rotor. The use of the former for the coil allows multiturn coils of a well-defined area to be reliably produced.
The data in Fig. 1 show that there is a slowly varying background signal resulting from the magnetization of the rotor. As the direction of the field sweep is changed so is the sign of the inducing electromotive force and hence the ECs reverse polarity. This allows them to be easily distinguished from both the magnetization of the rotor and the equilibrium magnetization (de Haas-van Alphen effect) of the 2DES.

The experiments were performed on both bulk- and $\delta$-modulation-doped $\mathrm{GaAs} /(\mathrm{Al}, \mathrm{Ga}) \mathrm{As}$ heterostructures containing 2DESs and on a SiGe heterostructure containing a two-dimensional hole system (2DHS). The transport properties of these samples are outlined in Table I.

\section{EXPERIMENTAL RESULTS}

In our experiments the analogue of a traditional, transport current vs voltage curve is an induced magnetic moment, $M$, vs time rate of change of field, $\partial B / \partial t$, curve, Fig. 1 (inset). The magnitude of the induced magnetic moments saturates to some value, $M_{s}$, at sufficiently fast sweep rates, and this saturation is attributed to the breakdown of the IQHE. ${ }^{14}$ Whereas in transport measurements it is $I_{x}$ that is fixed and an increase in $\rho_{x x}$ is indicative of the breakdown of the IQHE, as we shall show below, in our experiments the saturation of the induced magnetic moment is associated with an increase in $\sigma_{x x}$, and hence in a saturation of the induced currents.

$M_{s}$ is also dependent on temperature. Initial measurements found that this temperature dependence was very different for, empirically, "high"- and "low"-mobility samples. ${ }^{15}$ The range of sample mobilities used in this work has shown that crossover between the two types of behavior occurs around $\mu=75 \mathrm{~m}^{2} \mathrm{~V}^{-1} \mathrm{~s}^{-1}$ in GaAs 2DESs. The results presented here will be restricted to low-mobility (LM) samples, the temperature dependence of which are shown in Fig. 2, although more will be said about high-mobility samples in later sections.

The temperature dependences of the saturation value of the induced magnetic moment, $M_{s}(T)$, of all of the LM samples have the same general form and fit well to the linear relation

TABLE I. Sample details with their measured transport properties and extracted $g$-factors.

\begin{tabular}{lccccc}
\hline \hline Sample name & Grown at & $\begin{array}{c}\text { Carrier density } \\
\left(\times 10^{15} \mathrm{~m}^{-2}\right)\end{array}$ & $\begin{array}{c}\text { Mobility } \\
\left(\mathrm{m}^{2} \mathrm{~V}^{-1} \mathrm{~s}^{-1}\right)\end{array}$ & $\begin{array}{c}\text { Material } \\
\text { and system }\end{array}$ & $g^{*}$ \\
\hline T73 & Cambridge & 4.40 & 50.0 & GaAs 2DES & 4.90 \\
T151 & Cambridge & 1.83 & 72.5 & GaAs 2DES & 7.28 \\
NU762 & Nottingham & 3.60 & 27.0 & GaAs 2DES & 6.43 \\
HCO-198 & Niels Bohr & 1.45 & 30.0 & GaAs 2DES & $6.96^{\mathrm{a}}$ \\
$31 / 17$ & Warwick & 1.84 & 1.1 & SiGe 2DHS & $\mathrm{n} / \mathrm{a}$ \\
T393 & Cambridge & 1.02 & 100 & GaAs 2DES & $\mathrm{n} / \mathrm{a}$ \\
\hline \hline
\end{tabular}

${ }^{\mathrm{a}}$ For sample HCO-198 only $\nu=1$ and 2 were sufficiently well resolved to enable analysis, the value for the $g$-factor is obtained by fitting through the origin and hence this value is likely to be significantly less accurate than the other data in the table. For sample $31 / 17$ only $\nu=1$ was analyzed and so no $g$-factor could be extracted and the analysis is inappropriate for sample T393. 


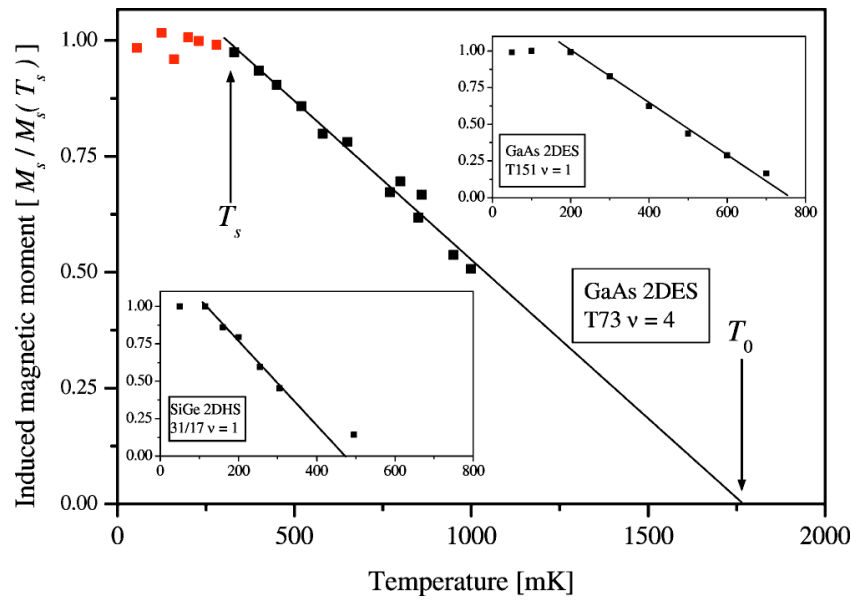

FIG. 2. The temperature dependence of the saturation magnetic moment at $\nu=4$ in sample T73. The linear trend, $M_{s}(T)=M_{0}-\alpha T$, and the values of $T_{s}$ and $T_{0}$ are indicated. The two insets demonstrate the similarity in behavior of both a two-dimensional electron system (top) and a two-dimensional hole system (bottom).

$$
M_{s}(T)=M_{0}-\alpha T \text {. }
$$

Here $M_{0}$ and $\alpha$ are constants that may depend on both the sample and the filling factor. In addition to this temperature dependence there may also be a low temperature cutoff of $M_{s}(T)$, i.e., $M_{s}(T)=$ constant, below some temperature $T_{s}$ as indicated in Fig. 2. The exact value of $T_{s}$ depends on sample density and filling factor (increasing with the energy gap) but is typically $\sim 300 \mathrm{mK}$.

The similarity between the results for the 2DES samples and the 2DHS sample is evident in Fig. 2.

\section{THEORY}

The experiments reveal several features that cannot be explained by any previous theory of the IQHE breakdown:

(1) The induced magnetic moments are much larger in low-mobility samples than in high-mobility ones.

(2) ECs exist only at low temperatures (typically, up to $1 \mathrm{~K}$ ), around two orders of magnitude below the cyclotron energy (which is approximately $20 \mathrm{~K} \mathrm{~T}^{-1}$ in GaAs 2DESs).

(3) In LM samples, the temperature dependence of $M_{s}$ is best fitted by a descending straight line; in some cases, a "cut-off" at low temperatures is observed.

(4) All the above features are apparently insensitive to the polarity of charge carriers and the chemical composition of the structure. Similar behavior has been observed for electrons in a GaAs/ $(\mathrm{Al}, \mathrm{Ga})$ As heterostructure and for holes in a SiGe one (Fig. 2).

To explain these observations we first consider a diskshaped sample of radius $R$. The induced magnetic moment, $M$, can be written as the integral of the tangential component of the current density, $j_{\varphi}(r)$, over the sample area,

$$
M=\pi \int_{0}^{R} j_{\varphi}(r) r^{2} d r=\pi \int_{0}^{R} \sigma_{x y} E_{r}(r) r^{2} d r,
$$

where $E_{r}(r)$ is the radial component of the in-plane electric field. Here we have used the fact that in the IQHE regime $j_{\varphi} \approx \sigma_{x y} E_{r}$.
Thus, to calculate the magnetic moment one has to find the radial electric field throughout the sample. This electric field is created by charges which are redistributed within the sample in such a way that the sample as a whole remains electrically neutral. As noticed by Dyakonov ${ }^{12}$ in twodimensional systems these charges cannot be concentrated at the edges, but must be distributed over the plane. In contactless experiments with disk-shaped samples, the validity of this statement is evident: there are simply no edge states at the center of the sample where some excess charge (positive or negative) could be placed.

The excess or deficit of charge carriers in the 2DES plane results in a shift of the Fermi-level, $\varepsilon_{F}$, from its initial position $(\varepsilon=0)$ midway between neighboring LLs. The probability of thermal activation of a charge carrier (an electron to the lowest empty level, or a hole to the uppermost filled level) is proportional to

$$
\begin{aligned}
P(\Delta n) \propto & \exp \left[-\left(\varepsilon_{0} / 2-\varepsilon_{F}(\Delta n) / k_{B} T\right)\right]+\exp \left[-\left(\varepsilon_{0} / 2\right.\right. \\
& \left.\left.+\varepsilon_{F}(\Delta n) / k_{B} T\right)\right],
\end{aligned}
$$

where $\varepsilon_{0}$ is the energy between the uppermost filled and the lowest empty electron levels in an idealized 2DES. As long as $|\Delta n|$ is small, $P(\Delta n)$ is a very small number and consequently $\sigma_{x x}$ is close to zero. As $|\Delta n|$ increases one of the exponentials increases, and the other decreases so that it can be neglected. This results in an exponential dependence of the longitudinal conductivity $\sigma_{x x}$ on $\varepsilon_{F}(|\Delta n|)$. As a consequence, $\sigma_{x x}$ should demonstrate a "threshold"-type behavior: there is no mobile charge until the exponent is greater than some critical value; above this value, the conductivity is high enough to provide charge relaxation, so that the radial field, $E_{r}$, cannot be sustained.

This threshold condition can be written as

$$
\exp \left(-\frac{\varepsilon_{0} / 2-\left|\varepsilon_{F}(\Delta n)\right|}{k_{B} T}\right)=C,
$$

where $C$ is a small number. Using (3) we find the threshold value of $|\Delta n|$ from

$$
\Delta n_{C}(T)=\int_{0}^{\varepsilon_{F}} \rho(\varepsilon) d \varepsilon=\int_{0}^{\varepsilon_{0} / 2+k_{B} T \ln C} \rho(\varepsilon) d \varepsilon,
$$

where $\rho(\varepsilon)$ is the density of states (DOS) for localized electrons. This result allows the construction of the electric field distribution in the sample which would correspond to the saturation of the magnetic moment in the IQHE regime. Indeed, the threshold behavior of conductivity allows two situations: (i) $|\Delta n|<\Delta n_{C}(T)$ at any $E_{r}$ or (ii) $|\Delta n|>\Delta n_{C}(T)$ at $E_{r}=0$. As we are interested in charge distributions that would provide maximum possible $E_{r}$, we construct it on the basis of condition (i), using, however, condition (ii) in small parts of the sample to provide self-consistency of the solution.

The intuitive first approximation for the charge distribution which gives the maximum possible value of $M$ is the following: $+e \Delta n_{C}(T)$ at $r<R / \sqrt{2}$ and $-e \Delta n_{C}(T)$ at $R / \sqrt{2}<r<R$ (or the same with the opposite sign, depending on the direction of the magnetic-field sweep). This distribution provides overall neutrality of the sample, while maxi- 


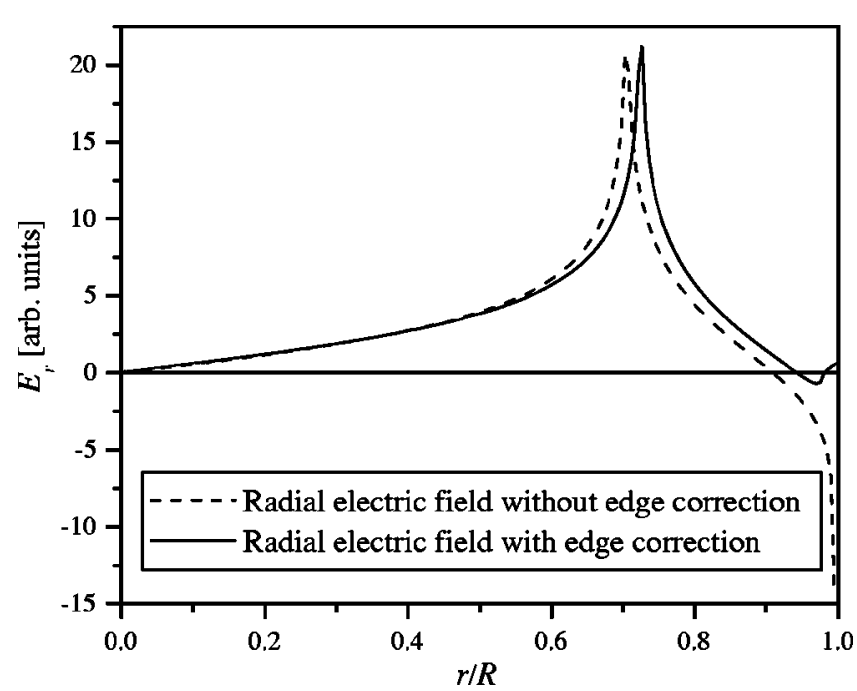

FIG. 3. The calculated radial electric field profile across the sample when the sweeping magnetic field is applied. The data shown as a dashed line ignore the charge build-up at the sample edge which is accounted for in the data represented by the solid line.

mum possible charge is moved. The electric field and potential created by this charge distribution in the sample plane can be expressed in terms of elliptic integrals $E$ and $K$,

$$
\begin{gathered}
E_{r}(r)= \pm e \Delta n_{C}[2 F(r, R / \sqrt{2})-F(r, R)], \\
U(r)= \pm e \Delta n_{C}[-2 G(r, R / \sqrt{2})+G(r, R)],
\end{gathered}
$$

where $\quad F(r, a)=\left[2 K(2 \sqrt{r a} /(r+a))\left(a^{2}+r^{2}\right)-2 E(2 \sqrt{r a} /(r\right.$ $\left.+a))(a+r)^{2}\right] / r(r+a)$ and $G(r, a)=2 E(2 \sqrt{r a} /(r+a))(r-a)$ $-2 K(2 \sqrt{r a} /(r+a))(r+a)$ are, respectively, the in-plane electric field and potential of a uniformly charged disk with radius $a$ and unit charge density. This result has been plotted against $r / R$ in Fig. 3 as a dashed line.

One can see that there are two singularities in the dependence of $E_{r}$ on radius, corresponding to the abrupt changes of the charge density at $r=R / \sqrt{2}$ and $r=R$. Both are integrable, so that there are no discontinuities of the electrostatic potential. However, negative values of the electric field near the edge are obviously nonphysical: they correspond to the current being directed opposite to the circulating electric field. The "wrong" direction of the electric field indicates that, in fact, more charge can be placed in this region. Indeed, a charge density exceeding $\Delta n_{C}$ would create a nonzero longitudinal conductivity, however the electric field would push the charge against the sample edge and it would not be able to escape. As a result, charge can accumulate near the edge until it completely screens the electric field, in accordance with condition (ii). The correct charge and field distribution can be recovered by guessing a coordinate dependence for the charge density near the edge such that it would make the electric field zero in that region. The solid line in Fig. 3 illustrates that this can indeed be done by placing an additional charge density of

$$
-e \delta n=-e \Delta n_{C}(T)[\sqrt{b /(R-r)}-1]
$$

into a narrow strip of width $b \approx 0.02 R$ along the edge. This eliminates the singularity and makes the field in this region close to zero.

Accordingly, the charge density now changes sign not at $r=R / \sqrt{2}$, but at $r=(R+b) / \sqrt{2}$, hence there is a shift of the other singularity towards larger $r$. Further refinement of the dependence of $E_{r}$ on $r$ is possible, but is not actually needed since the contribution of a small current in a narrow region to the total magnetic moment (2) is negligible.

The potential profile shown in Fig. 3 represents the most extreme case attainable within our model and would correspond to a system at the point of breakdown. The calculated radial field might well be modified by the presence of impurities and/or inhomogeneties and it is likely that the exact position of the maximum Hall field will also depend on the precise value of the filling factor. Indeed, within our model, we expect that at $\nu$ slightly different from an integer the threshold values of the accumulated charge density, $\Delta n_{C}$, would become different for positive and negative charge (due to the difference in energy between the position of the new quasi-Fermi energy and the extended states in the higher and lower LLs). This should result in a shift of the potential drop from $R / \sqrt{2}$ towards either the center, or the edge of the sample, accompanied by an overall decrease of the electric field and the corresponding induced current. These changes in the potential profile may be some of the factors determining the shape of the induced magnetic moment peak as a function of the magnetic field.

There have been experiments which have used atomic force microscopy techniques to map out the potential profile in contacted Hall bars and Corbino disks. ${ }^{16}$ However, these experiments were performed at low currents, well away from breakdown, and so do not correspond to the situation described here.

As the charge density over all the sample, and therefore $E_{r}$, is proportional to $\Delta n_{C}$, the saturation value of the magnetic moment, $M_{s}$, according to (2), is

$$
M_{s}=\Lambda \pi \sigma_{x y} e \Delta n_{C}(T) R^{3},
$$

where $\Lambda$ is a dimensionless constant which appears from the evaluation of the integral; our numerical calculations give $\Lambda \approx 1.1$. Thus, the temperature dependence of $M_{s}$ is the same as that for $\Delta n_{C}(T)$. According to (4), $\Delta n_{C}$ is the number of localized electron states within the energy range from zero (halfway between the filled and empty levels) and $\varepsilon_{0} / 2$ $+k_{B} T \ln C$ (note that $\ln C$ is negative). $\Delta n_{C}(T)$ vanishes at a temperature $T_{0}$, given by $T_{0}=\varepsilon_{0} / 2 k_{B}|\ln C|$. As $T \rightarrow 0, \Delta n_{C}(T)$ is one-half of the total number of localized states per unit area between the Landau levels. The behavior of $\Delta n_{C}(T)$ at $T<T_{0}$ is derived from the specific energy dependence of the density of states. Notably, if the DOS is approximately constant everywhere except in the vicinity of LLs, $\rho(\varepsilon)=\rho_{0}$, as one can expect for samples with strong disorder, ${ }^{17,18}$ the temperature dependence of $M_{s}$ is linear, 


$$
M_{s}=\Lambda \pi \sigma_{x y} e R^{3}\left(\varepsilon_{0} / 2-k_{B} T|\ln C|\right) \rho_{0}
$$

in agreement with experimental results, as shown above, for LM samples. As mentioned above, the Hall electric field assumed in this calculation may be unrealistic, in particular it might well be modified by the presence of impurities and/or inhomogeneties. Any such modification may influence the numerical constant $\Lambda$ in (7) but would not alter the central result of the paper: the linear temperature dependence of $M_{s}$.

One can see from (7) that $M_{s}$ is proportional to the density of localized states. Therefore, the more disordered the sample, the higher the current densities it can sustain.

However, the radial electric field will eventually reach values comparable to the quasielastic inter-Landau-level scattering (QUILLS) critical field. ${ }^{19}$ In the QUILLS model electrons scatter from the highest occupied ( $n$ th), to the adjacent empty $(n+1$ th) LL, conserving energy due to the Hall electric field. In their paper, Eaves and Sheard prohibited scattering between LLs of opposite spin, but we will discuss possible refinements to this model in a later section. The energy separation of the levels in the absence of a Hall field is $\hbar \omega_{c}$ where $\omega_{c}$ is the cyclotron frequency. The distance over which the electron may scatter is constrained by the need for wave function overlap between the initial and final states. Thus the Hall field must provide an energy difference of $\hbar \omega_{c}$ in this distance. The critical field is therefore given by

$$
E_{Q}=\frac{\hbar \omega_{c}}{e l_{B}\left([2 n+1]^{1 / 2}+[2 n+3]^{1 / 2}\right)} .
$$

Here the extent of the wave function is approximated by the size of semiclassical cyclotron orbits with $l_{B}$, the magnetic length, given by $l_{B}=(\hbar / e B)^{1 / 2}$.

This new mechanism will prevent $M_{s}$ from growing further and results in a saturation of $M_{s}$ as a function of temperature as $T$ approaches zero since the QUILLS process does not depend strongly on temperature. Such an effect is indeed observed, as shown in Fig. 2.

The QUILLS condition should first be met near $r=(R+b) / \sqrt{2}$, where the electric field is highest. The QUILLS condition in this case reads

$$
\Delta U=U\left(\frac{(R+b)}{\sqrt{2}}-\frac{\eta}{2} l_{B}\right)-U\left(\frac{(R+b)}{\sqrt{2}}+\frac{\eta}{2} l_{B}\right)=\hbar \omega_{c} / e,
$$

where $U(r)$ is the electrostatic potential, $\eta=\sqrt{2 n+1}$ $+\sqrt{2 n+3}$ and $n$ is the index of the uppermost filled LL. Expansion of $U(\mathrm{r})$ near the singularity give.

$$
\Delta U=\Delta n_{C} 4 e \eta l_{B} \ln \left(2 R / \eta l_{B}\right) .
$$

Thus, the charge density corresponding to the onset of QUILLS at the peak of the electric field, shown in Fig. 3 is $e \Delta n_{Q}=E_{Q} / 4 \ln \left(2 R / \eta l_{B}\right)$, where $E_{Q}=\hbar \omega_{c} / e \eta l_{B}$ is the value of uniform electric field at which QUILLS is expected to occur. The corresponding magnetic moment of the sample is

$$
M_{Q}=\Lambda \pi \sigma_{x y} \frac{E_{Q}}{4 \ln \left(2 R / \eta l_{B}\right)} R^{3} .
$$

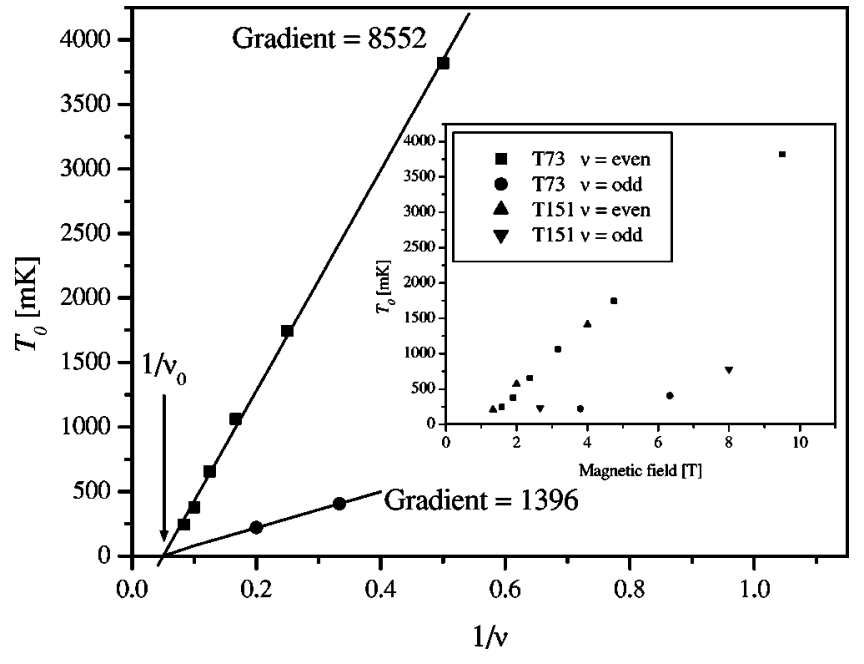

FIG. 4. $T_{0}$ plotted against $1 / \nu$ for sample T73. The odd and even filling factors have different gradients, as indicated. The inset shows data for both T73 and T151 plotted against magnetic field, the even $\nu$ data then have the same gradient (within experimental error). The difference between the odd $\nu$ data reflects the different $g$-factors in these samples.

\section{DISCUSSION}

The theory presented above (7) gives a temperature dependence of $M_{s}$ which agrees with the experimental data, Fig. 2. From comparison with experiment some information can be extracted.

\section{A. $g$-factors}

The cut-off on the temperature axis $T_{0} \quad\left(k_{B} T_{0}\right.$ $\left.=\varepsilon_{0} /(2|\ln C|)\right)$ measured at a range of filling factors, provides information about $\varepsilon_{0}$ as a function of magnetic field. Although $\ln C$ is not known, one can compare the dependences for even and odd filling factors: for even $\nu, \varepsilon_{0}=\hbar \omega_{c}$; for odd $\nu, \varepsilon_{0}=g^{*} \mu_{B} B$. As $\ln C$ should be same for both cases, this provides a way to determine the effective g-factor, $g^{*}$, which is believed, both theoretically ${ }^{20}$ and experimentally, ${ }^{21}$ to be much greater than the single-electron $g$-factor, as a result of many-body effects. Figure 4 shows $T_{0}$ plotted against $1 / \nu$ for sample T73.

Separate linear fits to the data for odd filling factors and even filling factors show different gradients, as expected from the difference in the nature of the energy gap, and a positive intercept on the $1 / \nu$ axis.

The fact that $T_{0}$ goes to zero at finite $\nu$ has the appealing physical interpretation that $\varepsilon_{0}$ is, in fact, a mobility gap rather than the LL separation. The value of the intercept then indicates the filling factor at which there would be no mobility gap between LLs even at $T=0$, i.e., it is related to the width of the LLs. Similar results have been obtained for even $\nu$ from equilibrium magnetization measurements ${ }^{22}$ and, for odd $\nu$, by transport measurements ${ }^{23}$ by other authors. The fact that this intercept is the same for both odd and even filling factors is surprising but has been shown in all the samples for which sufficient filling factors to plot data as in Fig. 4 were observed. The value of $\nu_{0} / n_{e}$ may provide some 


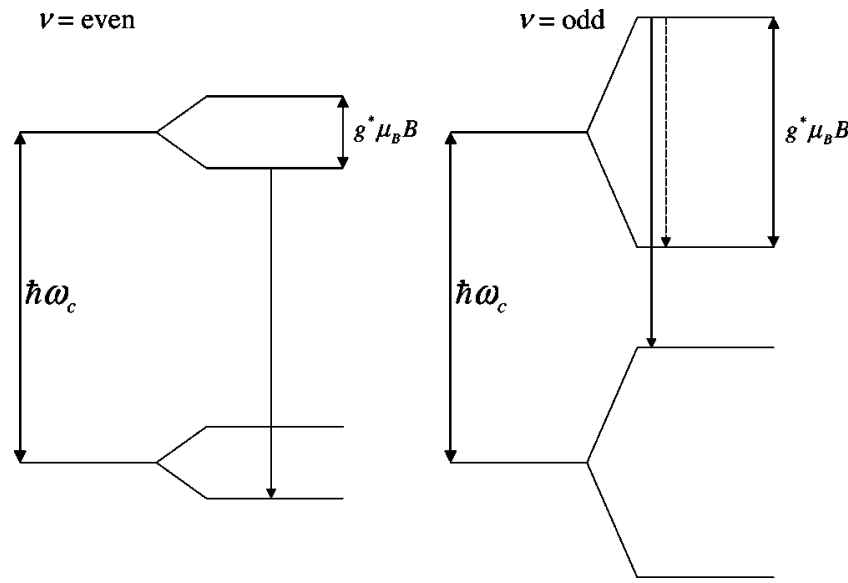

FIG. 5. A sketch of possible inter-Landau level transitions which both conserve spin (solid arrows) and allow spin-flip transitions (dashed arrows).

measure of the intrinsic sample quality but due to the fact that not all of the samples measured had a sufficient number of filling factors that were well enough resolved to enable analysis, no obvious correlation between $\nu_{0} / n_{e}$ and the sample mobility could be found.

From the ratio, $\beta$, of the gradients of the linear fits for odd filling factors to that for even the effective $g$-factor can be extracted as

$$
g^{*}=2\left(\frac{m}{m^{*}}\right) \beta .
$$

For sample T73 $g^{*}=4.9$, exchange-enhanced by a factor of 11 from its bare value which is consistent with results obtained on similar samples using other experimental techniques. $^{24}$ The extracted values for the other samples are included in Table I.

\section{B. QUILLS}

In the LM samples a low-temperature cut-off to the maximum size of $M_{s}$ is observed below $\sim 300 \mathrm{mK}$, Fig. 2. This may be due to the maximum electric field in the sample becoming large enough to cause IQHE breakdown by QUILLS (8).

As shown above the energy gaps between extended states are different for odd and even LLs and so it would be appropriate to modify (8) to account for this. Hence the initial restriction in the QUILLS model that all transitions must conserve spin should be relaxed. The possible QUILLS transitions for both odd and even LLs are sketched in Fig. 5. Since $g$-factor enhancement is an oscillatory function of the LL filling factor, ${ }^{20}$ for even $\nu$ the energy gap is always $\approx \hbar \omega_{c}$, for odd $\nu$ there are two possibilities: $\approx \hbar \omega_{c}$ if spin is conserved; $g^{*} \mu_{B} B$ if it is not. In addition, if spin is not conserved the denominator of (8) should be modified to account for the fact that the LL index, $n$, does not change in these transitions. This correction will be most significant at high fields ( $\operatorname{small} n$ ).

The calculated maximum magnetic moments subject to the QUILLS condition (11) can be compared with experi-

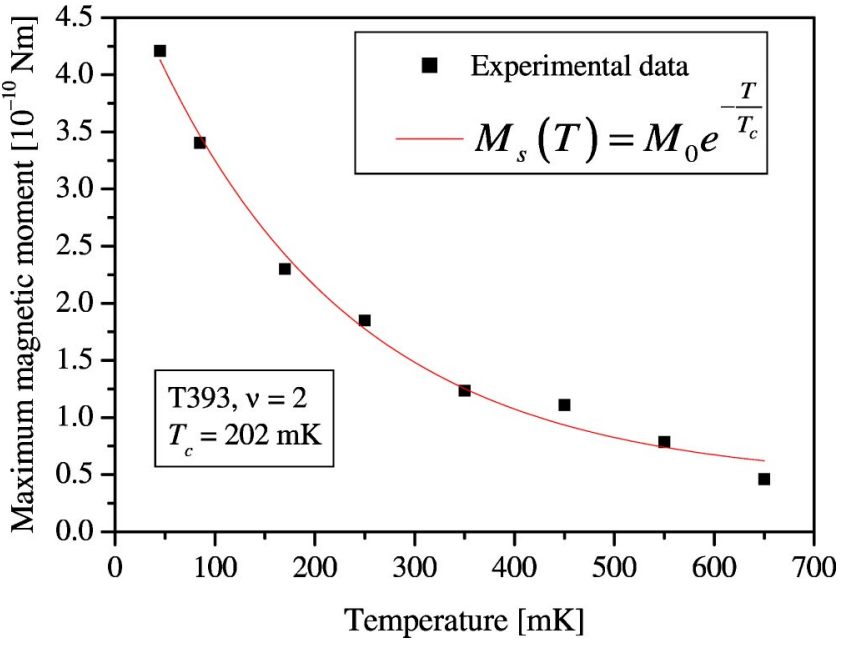

FIG. 6. The temperature dependence of the saturation magnetic moment in the high-mobility sample T393. The experimental data have been fitted to a simple exponential dependence.

ment in samples where low-temperature cut-offs of $M_{s}(T)$ are observed. We find that that the odd filling factor experimental data are closer to theory if spin-flip transitions are allowed, in these calculations the effective $g$-factors for each sample, as quoted in Table I, were used. For both odd and even filling factors we find

$$
M_{\mathrm{MAX}}^{\text {Theory }} / M_{\mathrm{MAX}}^{\text {Experiment }} \approx 4 .
$$

Although the exact ratio in (12) depends on both the sample and filling factor there is reasonable agreement between experiment and theory with no fitting parameters. It is likely that these results could be improved by adding a scattering rate to the QUILLS condition, as in the breakdown model, to account for the possibility of LL transitions occurring before (8) is satisfied. Further, accounting for nonuniform samples, the finite width of the LLs, considering the exact sample geometry and refining the model for the radial charge distribution could all improve the agreement between experiment and theory. Such refinements would, however, not change the conclusion that induced currents in LM 2DESs and 2DHSs at low temperatures are limited by QUILLS. At higher temperatures $M_{s}$ saturates due the bulk charge redistribution which increases $\sigma_{x x}$.

\section{High-mobility samples}

High-mobility (HM) samples, perhaps nonintuitively, support smaller induced currents, with a much stronger temperature dependence than the low-mobility ones. ${ }^{15}$ There is also no sign of a low-temperature cutoff of $M_{s}$ for $T>50 \mathrm{mK}$, Fig. 6. Although the model described above cannot be used to say anything quantitative about HM samples at present the general features of their behavior can be explained.

High mobility samples are expected to have a lower density of states between LLs than LM ones. ${ }^{25}$ According to (8) the size of $M_{s}$ is directly proportional to $\rho_{0}$ and hence a smaller induced current is expected. The fact that there is no low-temperature cutoff in the $M_{s}(T)$ data means that the Hall 
electric field in the sample never reaches a large enough value to cause QUILLS. Experiments at ultralow temperatures may, however, observe QUILLS breakdown in HM samples.

The fact that the DOS between LLs is lower also means that a given charge redistribution will require the Fermi level to move further towards a mobility edge in HM samples as compared to LM ones. This in turn will result in a stronger temperature dependence of $M_{s}$. The fact that the temperature dependence is no longer linear can also be understood using this picture: since the Fermi level is moving further from a minimum in the DOS towards a LLs peak it is sampling more of the DOS. As the Fermi energy moves further from the midpoint between LLs the DOS is likely not to be constant, and so the temperature dependence of $M_{s}$ actually reflects the energy dependence of the DOS. The exponential form shown in Fig. 5 is consistent with trial forms of the DOS previously used to fit equilibrium magnetization data. ${ }^{26}$ It is possible that future work could use $M_{s}(T)$ data to determine the functional form of the DOS between LLs in both the IQHE and FQHE regimes.

We also note that transport experiments have shown a transition between two types of IQHE breakdown behavior: ${ }^{27,28}$ here the critical current required for breakdown scales either linearly or sublinearly with the sample width. The transition from linear to sublinear behavior was achieved by illumination of the sample when cold and the change was explained in terms of potential fluctuations in the sample. It is such potential fluctuations which define the DOS between LLs and so, in our work, we may expect to see a transition from LM-type behavior (large ECs with a linear temperature dependence) to HM-type behavior (small ECs with an approximately exponential temperature dependence) after illumination of a LM sample when cold. Exactly this effect has been observed in sample T151, ${ }^{29}$ where illumination raised its number density and mobility to $1.83 \times 10^{15} \mathrm{~m}^{-2}$ and $120 \mathrm{~m}^{2} \mathrm{~V}^{-1} \mathrm{~s}^{-1}$, respectively.

\section{CONCLUSIONS}

Induced currents have been used to study the temperature dependence of the breakdown of the IQHE in low-mobility samples. We find that the maximum magnetic moment which it is possible to induce in these samples decreases linearly with increasing temperature. To explain these results we have developed a model which accounts for the highly nonlinear dependence of the sample conductivity on the charge redistribution in the 2DES plane. The temperature dependence is related to the energy separation between extended states and the DOS between LLs, these facts can be used to extract a value for the effective $g$-factor by comparing spin-split energy gaps with the cyclotron energy gap.

In addition, at low enough temperatures, the induced currents can become sufficiently large to cause IQHE breakdown by QUILLS. There is reasonable agreement between theory and experiment with no fitting parameters and it is likely that this agreement could be improved by refining the model.

The way in which this analysis could be extended to highmobility samples has also been discussed and provides a qualitative explanation for the different behavior observed in these systems. The possibility for obtaining the DOS between LLs in high-mobility samples from the temperature dependence of breakdown has been outlined.
*Present address: A. F. Ioffe Physico-Technical Institute, St. Petersburg, Russia.

'Present address: School of Physics, University of New South Wales, Australia.

${ }^{1}$ K. von Klitzing, G. Dorda, and M. Pepper, Phys. Rev. Lett. 45, 494 (1980).

${ }^{2}$ G. Ebert, K. von Klitzing, K. Ploog, and G. Weimann, J. Phys. C 16, 5441 (1983).

${ }^{3}$ L. Bliek, G. Hein, D. Jucknischke, V. Kose, J. Miemeyer, G. Weimann, and W. Schlapp, Surf. Sci. 196, 156 (1988).

${ }^{4}$ O. Heinonen, P. L. Taylor, and S. M. Girvin, Phys. Rev. B 30, 3016 (1984).

${ }^{5}$ P. Středa and K. von Klitzing, J. Phys. C 17, L483 (1984).

${ }^{6}$ S. Komiyama, T. Takamasu, S. Hiyamizu, and S. Sasa, Solid State Commun. 54, 479 (1985).

${ }^{7}$ S. Kawaji, Semicond. Sci. Technol. 11, 1546 (1996).

${ }^{8}$ O. P. Hansen, A. Kristensen, and H. Bruus, Superlattices Microstruct. 8, 365 (1990).

${ }^{9}$ V. T. Dolgopolov, A. A. Shashkin, N. B. Zhitenev, S. I. Dorozhkin, and K. von Klitzing, Phys. Rev. B 46, 12560 (1992).

${ }^{10}$ For a review, see G. Nachtwei, Physica E (Amsterdam) 4, 79 (1999), and references therein.

${ }^{11}$ C. L. Jones, A. Usher, M. Elliott, W. G. Herrenden-Harker, A.
Potts, R. Shepherd, T. S. Cheng, and C. T. Foxon, Solid State Commun. 97, 763 (1996).

${ }^{12}$ M. I. Dyakonov, Solid State Commun. 78, 817 (1991).

${ }^{13}$ C. L. Jones, A. Usher, M. Elliott, W. G. Herrenden-Harker, A. Potts, R. Shepherd, T. S. Cheng, and C. T. Foxon, Solid State Commun. 95, 409 (1995); A. J. Matthews, A. Usher, and C. D. H. Williams, Rev. Sci. Instrum. 75, 2672 (2004).

${ }^{14}$ J. P. Watts, A. Usher, A. J. Matthews, M. Zhu, M. Elliott, W. G. Herrenden-Harker, P. R. Morris, M. Y. Simmons, and D. A. Ritchie, Phys. Rev. Lett. 81, 4220 (1998).

${ }^{15}$ A. J. Matthews, J. P. Watts, M. Zhu, A. Usher, M. Elliott, W. G. Herrenden-Harker, P. R. Morris, M. Y. Simmons, and D. A. Ritchie, Physica E (Amsterdam) 6, 140 (2000).

${ }^{16}$ E. Ahlswede, J. Weis, K. von Klitzing, and K. Eberl, Physica E (Amsterdam) 12, 165 (2002).

${ }^{17}$ E. Gornik, R. Lassnig, G. Strasser, H. L. Störmer, A. C. Gossard, and W. Eiegmann, Phys. Rev. Lett. 54, 1820 (1985).

${ }^{18}$ V. Sa-yakanit, N. Choosiri, and H. R. Glyde, Phys. Rev. B 38, 1340 (1988).

${ }^{19}$ L. Eaves and F. W. Sheard, Semicond. Sci. Technol. 1, 346 (1986).

${ }^{20}$ For a review, see.T. Ando, A. B. Fowler, and F. Stern, Rev. Mod. Phys. 54, 437 (1982). 
${ }^{21}$ R. J. Nicholas, R. J. Haug, K. von Klitzing, and G. Weimann, Phys. Rev. B 37, 1294 (1988).

${ }^{22}$ M. P. Schwarz, M. A. Wilde, S. Groth, D. Grundler, Ch. Heyn, and D. Heitmann, Phys. Rev. B 65, 245315 (2002).

${ }^{23}$ D. R. Leadley, R. J. Nicholas, J. J. Harris, and C. T. Foxon, Phys. Rev. B 58, 13036 (1998).

${ }^{24}$ A. Usher, R. J. Nicholas, J. J. Harris, and C. T. Foxon, Phys. Rev. B 41, 1129 (1990).

${ }^{25}$ G. Nachtwei, O. Salchow, C. Breitlow, A. Jaeger, and H. Kruger,
Surf. Sci. 250, 243 (1991).

${ }^{26}$ A. Potts, R. Shepherd, W. G. Herrenden-Harker, M. Elliott, C. L. Jones, A. Usher, G. A. Jones, D. A. Ritchie, E. H. Linfield, and M. Grimshaw, J. Phys.: Condens. Matter 8, 5189 (1996).

${ }^{27}$ N. Q. Balaban, U. Meirav, and Hadas Shtrikman, Phys. Rev. B 52, R5503 (1995).

${ }^{28}$ T. Sanuki, K. Oto, S. Takaoka, K. Murase, and K. Gamo, Solid State Commun. 117, 343 (2001).

${ }^{29}$ A. J. Matthews, Ph.D. thesis, University of Exeter, 2001. 